\title{
Global Attractor of Two-Dimensional Strong Damping KDV Equation and Its Dimension Estimation
}

\author{
Cheng Zhang, Guoguang Lin \\ Mathematical of Yunnan University, Kunming, China \\ Email: zhangcheng198910@126.com, gglin@ynu.edu.cn
}

Received October 10, 2013; revised November 10, 2013; accepted November 17, 2013

Copyright (C) 2014 Cheng Zhang, Guoguang Lin. This is an open access article distributed under the Creative Commons Attribution License, which permits unrestricted use, distribution, and reproduction in any medium, provided the original work is properly cited. In accordance of the Creative Commons Attribution License all Copyrights (C) 2014 are reserved for SCIRP and the owner of the intellectual property Cheng Zhang, Guoguang Lin. All Copyright (C) 2014 are guarded by law and by SCIRP as a guardian.

\section{ABSTRACT}

Firstly, a priori estimates are obtained for the existence and uniqueness of solutions of two dimensional KDV equations, and prove the existence of the global attractor, finally geting the upper bound estimation of the Hausdorff and fractal dimension of attractors.

\section{KEYWORDS}

\section{KDV Equation; Strongly Damped; Existence; Global Attractor; Dimension Estimation}

\section{Introduction}

Studies on the infinite dimension system with high dimension have obtained many achievements in recent years, such as [1-5]. In the paper [6,7]. The authors study the estimates of global attractor for one-dimensional KDV equation and its dimension. Based on these work, this paper further studies the global attractor of twodimensional KDV equations and its upper bound estimation of the Hausdorff and fractal dimension of attractors.

The following form 2D-KDV equation is studied in this paper

$$
\begin{aligned}
& u_{t}+u_{x x x}+\alpha u+\beta(u v)_{x}+\gamma \Delta^{2} u=f(x, y),(x, y) \in \Omega \\
& u_{x}(x, y ; t)=v_{y}(x, y ; t), \quad(x, y) \in \Omega \\
& u(x, y ; 0)=u_{0}(x, y), \quad(x, y) \in \Omega \\
& \left.u(x, y ; t)\right|_{\partial \Omega}=0,\left.\Delta u(x, y ; t)\right|_{\partial \Omega}=0,(x, y) \in \Omega
\end{aligned}
$$

where $\alpha, \beta, \gamma$ are positive constants. When $\alpha=\beta=\gamma=0$, the equation is the KDV equation.

The rest of this paper is organized as follows. In Section 2, we introduce basic concepts concerning global attractor. In Section 3, we obtain the existence of the uniqueness global attractor, which has fractal and Hausdorff dimension.

In this paper, $C$ denotes a positive constant whose value may change in different positions of chains of inequalities.

\section{Preliminaries}

Denoting by $|\cdot|_{L^{p}}$ the norm in $L^{p}(\Omega), 1 \leq p \leq \infty$, for simplicity, we denote by $|\cdot|$ and $|\cdot|_{\infty}$ the norm in the case $p=2$ and $p=\infty$, respectively. Suppose that $H=L^{2}(\Omega), H^{i}(\Omega)$ is a Hilbert space for the scalar 
product

$$
((\cdot, \cdot))_{H}^{i}=(\cdot, \cdot)+\sum_{j=1}^{i}\left(D^{j} \cdot, D^{j} \cdot\right), D=\frac{\partial}{\partial x} .
$$

According to the Poincare inequality and (1.2) we can get

$$
|v| \leq C_{1}|\nabla u|
$$

In fact,

$$
u_{x}=v_{y} \Rightarrow u_{x x}=v_{x y} \Rightarrow\left|u_{x x}\right| \leq\left|v_{x y}\right| \leq C|\Delta u| \Rightarrow\left|v_{x}\right| \leq C\left|v_{x y}\right| \leq C|\Delta u| \Rightarrow|v| \leq C_{1}|\nabla u|
$$

Now, we can do priori estimates for Equation (1.1)

Lemma 1. Assume that $f(x, y) \in L^{2}(\Omega), u_{0}(x, y) \in L^{2}(\Omega), \alpha>\frac{\beta^{2} C^{2}}{2 \gamma}$ then

$$
|u(x, y ; t)|^{2} \leq\left|u_{0}(x, y)\right|^{2} \mathrm{e}^{-\left(2 \alpha-\frac{\beta^{2} C^{2}}{\gamma}\right) t}+\frac{2 \gamma}{\beta^{2} C^{2}\left(2 \alpha-\frac{\beta^{2} C^{2}}{\gamma}\right)}|f|^{2}\left(1-\mathrm{e}^{-\left(2 \alpha-\frac{\beta^{2} C^{2}}{\gamma}\right) t}\right),
$$

Certainly there exist $t_{1}=t_{1}(\Omega)>0$, such that

$$
|u(x, y ; t)| \leq C_{2},
$$

Proof. We multiply $u$ for both sides of Equation (1.1), we obtain

$$
\left(u_{t}, u\right)+\left(u_{x x x}, u\right)+\alpha(u, u)+\beta\left((u v)_{x}, u\right)+\gamma\left(\Delta^{2} u, u\right)=(f, u),
$$

where $\left(u, u_{x x x}\right)=-\left(u_{x x x}, u\right)$, we have

$$
\begin{aligned}
& \left(u, u_{x x x}\right)=0, \\
& \beta\left|\left((u v)_{x}, u\right)\right|=\beta\left|\left(u v, u_{x}\right)\right| \leq \beta|u|_{\infty}|\nabla u||v| \leq \beta|u|_{\infty}|\nabla u|^{2} \leq \beta C|\Delta u||u| \leq \gamma|\Delta u|^{2}+\frac{\beta^{2} C^{2}}{4 \gamma}|u|^{2}, \\
& |(u, f)| \leq|u||f| \leq \frac{\beta^{2} C^{2}}{4 \gamma}|u|^{2}+\frac{\gamma}{\beta^{2} C^{2}}|f|^{2},
\end{aligned}
$$

Substituting (2.4)-(2.6) into (2.3) gets

$$
\frac{1}{2} \frac{\mathrm{d}}{\mathrm{d} t}|u|^{2}+\left(\alpha-\frac{\beta^{2} C^{2}}{2 \gamma}\right)|u|^{2} \leq \frac{\gamma}{\beta^{2} C^{2}}|f|^{2}
$$

Using the Growall inequality, we can get

$$
|u(x, y ; t)|^{2} \leq\left|u_{0}(x, y)\right|^{2} \mathrm{e}^{-\left(2 \alpha-\frac{\beta^{2} C^{2}}{\gamma}\right) t}+\frac{2 \gamma}{\beta^{2} C^{2}\left(2 \alpha-\frac{\beta^{2} C^{2}}{\gamma}\right)}|f|^{2}\left(1-\mathrm{e}^{-\left(2 \alpha-\frac{\beta^{2} C^{2}}{\gamma}\right) t}\right)
$$

Lemma 2. Assume that $f(x, y) \in H_{0}^{1}(\Omega), u_{0}(x, y) \in H_{0}^{1}(\Omega), \alpha>\frac{\beta^{2} C^{2}}{2 \gamma}$ then

$$
|\nabla u(x, y ; t)|^{2} \leq\left|\nabla u_{0}(x, y)\right| \mathrm{e}^{-2 \alpha t}+\frac{|\nabla f(x, y)|^{2}+2 \alpha C}{\alpha^{2}}\left(1-\mathrm{e}^{-2 \alpha t}\right),
$$


certainly, there also exist $t_{2}=t_{2}(\Omega)>0$, such that

$$
\varlimsup_{t \rightarrow \infty}|\nabla u(x, y ; t)|^{2} \leq C_{3},
$$

Proof. We take parts of the scalar product in $L^{2}$ of (1.1) with $-\Delta u$ :

$$
\left(u_{t},-\Delta u\right)+\left(u_{x x x},-\Delta u\right)+\alpha(u,-\Delta u)+\beta\left((u v)_{x},-\Delta u\right)+\gamma\left(\Delta^{2} u,-\Delta u\right)=(f,-\Delta u),
$$

where $\left(u_{x x x},-\Delta u\right)=\left(\Delta u, u_{x x x}\right)$, thus

$$
\begin{aligned}
& \left(u_{x x x},-\Delta u\right)=0, \\
& \beta\left|\left((u v)_{x},-\Delta u\right)\right|=\beta\left|\left(u v, \Delta u_{x}\right)\right| \leq \beta|u|_{\infty}|\nabla u||\nabla \Delta u|,
\end{aligned}
$$

Noticing

$$
\begin{aligned}
& |u|_{\infty} \leq C|\nabla \Delta u|^{\frac{1}{3}}|u|^{\frac{2}{3}}, \\
& |\nabla u| \leq C|\nabla \Delta u|^{\frac{1}{3}}|u|^{\frac{2}{3}},
\end{aligned}
$$

According to (12) and (13), Lemma 1 and Young inequality, we can obtain that

$$
\begin{aligned}
& \beta\left|\left((u v)_{x},-\Delta u\right)\right| \leq C|\nabla \Delta u|^{\frac{5}{3}}|u|^{\frac{4}{3}} \leq \gamma|\nabla \Delta u|^{2}+C, \\
& |(f,-\Delta u)| \leq|\nabla f||\nabla u| \leq \frac{1}{2 \alpha}|\nabla f|^{2}+\frac{\alpha}{2}|\nabla u|^{2},
\end{aligned}
$$

Using (2.10), (2.14) and (2.15), we can get

$$
\frac{1}{2} \frac{\mathrm{d}}{\mathrm{d} t}|\nabla u|^{2}+\frac{\alpha}{2}|\nabla u| \leq \frac{1}{2 \alpha}|\nabla f|^{2}+C
$$

Using Growall inequality, we have

$$
|\nabla u|^{2} \leq\left|\nabla u_{0}\right|^{2} \mathrm{e}^{-2 \alpha t}+\frac{|\nabla f|^{2}+2 \alpha C}{\alpha^{2}}\left(1-\mathrm{e}^{-2 \alpha t}\right)
$$

Lemma 3. Assume that $f(x, y) \in H_{0}^{2}(\Omega), u_{0}(x, y) \in H_{0}^{2}(\Omega), \alpha>\frac{\beta^{2} C^{2}}{2 \gamma}$ then

$$
|\Delta u(x, y ; t)|^{2} \leq\left|\Delta u_{0}(x, y)\right| \mathrm{e}^{-2 \alpha t}+\frac{|\Delta f|^{2}+2 \alpha C}{\alpha^{2}}\left(1-\mathrm{e}^{-2 \alpha t}\right),
$$

Thus there exists $t_{3}=t_{3}(\Omega)>0$, such that

$$
|\Delta u(x, y ; t)| \leq C_{4},
$$

Proof. We multiply $\Delta^{2} u$ for both sides of Equation (1.1), we obtain that

$$
\left(u_{t}, \Delta^{2} u\right)+\left(u_{x x x}, \Delta^{2} u\right)+\alpha\left(u, \Delta^{2} u\right)+\beta\left((u v)_{x}, \Delta^{2} u\right)+\gamma\left(\Delta^{2} u, \Delta^{2} u\right)=\left(f, \Delta^{2} u\right),
$$

where

Noticing

$$
\left(u_{x x x}, \Delta^{2} u\right)=0
$$

$$
|u|_{\infty} \leq C\left|\Delta^{2} u\right|^{\frac{1}{4}}|u|^{\frac{3}{4}}
$$




$$
|\Delta u| \leq C\left|\Delta^{2} u\right|^{\frac{1}{3}}|\nabla u|^{\frac{2}{3}}
$$

Using (2.20)-(2.21), we obtain that

$$
\beta\left|\left((u v)_{x}, \Delta^{2} u\right)\right| \leq \beta C|u|_{\infty}|\Delta u|\left|\Delta^{2} u\right| \leq C\left|\Delta^{2} u\right|^{\frac{19}{12}}\left|u^{\frac{3}{4}}\right||\nabla u|^{\frac{2}{3}},
$$

According to Lemma 1, Lemma 2 and Young inequality, we get that

$$
\begin{aligned}
& \beta\left|\left((u v)_{x}, \Delta^{2} u\right)\right| \leq \gamma\left|\Delta^{2} u\right|^{2}+C, \\
& \left|\left(f, \Delta^{2} u\right)\right| \leq|\Delta u||\Delta f| \leq \frac{\alpha}{2}|\Delta u|^{2}+\frac{1}{2 \alpha}|\Delta f|^{2},
\end{aligned}
$$

Substituting (2.19)-(2.24) into (2.18) gets

$$
\frac{1}{2} \frac{\mathrm{d}}{\mathrm{d} t}|\Delta u|^{2}+\frac{\alpha}{2}|\Delta u|^{2} \leq \frac{1}{2 \alpha}|\Delta f|^{2}+C
$$

Using the Growall inequality, we can get

$$
|\Delta u|^{2} \leq\left|\Delta u_{0}\right|^{2} \mathrm{e}^{-2 \alpha t}+\frac{|\Delta f|^{2}+2 \alpha C}{\alpha^{2}}\left(1-\mathrm{e}^{-2 \alpha t}\right)
$$

Lemma 4. Assume that $f(x, y) \in H_{0}^{2}(\Omega), u_{0}(x, y) \in H_{0}^{2}(\Omega), \alpha>\frac{\beta^{2} C^{2}}{2 \gamma}$ then

$$
|\nabla \Delta u(x, y ; t)| \leq \frac{Q}{t}
$$

here $Q$ and $\left|u_{0}\right|_{H_{0}^{2}},|f|_{H_{0}^{2}}$ have relations.

Proof. We multiply $t^{2} \Delta^{3} u$ for both sides of Equation (1.1), we obtain that

$$
\left(u_{t}, t^{2} \Delta^{3} u\right)+\left(u_{x x x}, t^{2} \Delta^{3} u\right)+\alpha\left(u, t^{2} \Delta^{3} u\right)+\beta\left((u v)_{x}, t^{2} \Delta^{3} u\right)+\gamma\left(\Delta^{2} u, t^{2} \Delta^{3} u\right)=\left(f, t^{2} \Delta^{3} u\right),
$$

we have

$$
\begin{aligned}
& \left(u_{t}, t^{2} \Delta^{3} u\right)=-\frac{1}{2} \frac{\mathrm{d}}{\mathrm{d} t}|t \nabla \Delta u|^{2}+\left|t^{\frac{1}{2}} \nabla \Delta u\right|^{2}, \\
& \gamma\left(\Delta^{2} u, t^{2} \Delta^{3} u\right)=-\gamma\left|t \nabla \Delta^{2} u\right|^{2}, \\
& \left(u_{x x x}, t^{2} \Delta^{3} u\right)=0 \\
& \left|\left(f, \Delta^{3} u\right)\right|=|\nabla f|\left|\nabla \Delta^{2} u\right| \leq \frac{\gamma}{6}\left|\nabla \Delta^{2} u\right|^{2}+\frac{3}{2 \gamma}|\nabla f|^{2}, \\
& \alpha\left|\left(u, \Delta^{3} u\right)\right| \leq \alpha|\nabla u|\left|\nabla \Delta^{2} u\right|^{2} \leq \frac{\gamma}{6}\left|\nabla \Delta^{2} u\right|^{2}+C, \\
& \beta\left|\left((u v)_{x}\right), \Delta^{3} u\right|=\left|\left(\nabla\left(u_{x} v+u v_{x}\right), \nabla \Delta^{2} u\right)\right| \leq C\left(3|\nabla u|_{\infty}|\Delta u|+|u|_{\infty}|\nabla \Delta u|\right)\left|\nabla \Delta^{2} u\right|,
\end{aligned}
$$

Noticing

$$
|u|_{\infty} \leq C|\Delta u|^{\frac{1}{2}}|u|^{\frac{1}{2}}
$$




$$
\begin{aligned}
& |\nabla u|_{\infty} \leq C|\Delta u|^{\frac{3}{4}}\left|\nabla \Delta^{2} u\right|^{\frac{1}{4}}, \\
& |\nabla \Delta u| \leq C|u|^{\frac{2}{5}}\left|\nabla \Delta^{2} u\right|^{\frac{3}{5}},
\end{aligned}
$$

Taking (2.33)-(2.35) into (2.32) and using Young inequality, we have

$$
\beta\left|\left((u v)_{x}, \Delta^{3} u\right)\right| \leq \frac{\gamma}{6}\left|\nabla \Delta^{2} u\right|^{2}+C,
$$

namely,

$$
\beta\left|\left((u v)_{x}, t^{2} \Delta^{3} u\right)\right| \leq \frac{\gamma}{6}\left|t \nabla \Delta^{2} u\right|^{2}+C
$$

Taking (2.27)-(2.37) into (2.26), we obtain

So, we get

$$
\frac{\mathrm{d}}{\mathrm{d} t}|t \nabla \Delta u|^{2}+\gamma|t \nabla \Delta u|^{2} \leq C|\nabla f|^{2}
$$

$$
|\nabla \Delta u| \leq \frac{Q}{t}
$$

From [8], we have

Theorem 2.1 Let $E$ be a Banach space, $\{S(t)\}$ are the semigroup operators. $S(t): E \rightarrow E, S(t) S(\tau)$ $=S(t+\tau), S(0)=I$, here $I$ is unit operator.Set $S(t)$ satisfy the following conditions:

1) $S(t)$ is bounded. namely $\forall R>0,|u|_{E} \leq R$, there exist a constant $C(R)$, such that $|S(t) u|_{E}$ $\leq C(R)(t \in[0,+\infty))$.

2) There exist a bounded absorbing set $B_{0} \subset E$, namely $\forall B \subset E$, there exist a constant $t_{0}$, such that $S(t) B \subset B_{0}\left(t>t_{0}\right)$.

3) When $t>0, S(t)$ is a completely continuous operator.

Then, the semigroup operators $S(t)$ exist a compact global attractor $A$.

\section{Global Attractor and Dimension Estimation}

\subsection{The Existence and Uniqueness of Solution}

Theorem 3.1 Assume that $f(x, y) \in H_{0}^{2}(\Omega)$ and $u_{0}(x, y) \in H_{0}^{2}(\Omega), \alpha>\frac{\beta^{2} C^{2}}{2 \gamma}$ there exists a unique solution

$$
u(x, y ; t) \in L^{\infty}\left(0, T ; H_{0}^{2}(\Omega)\right)
$$

Proof. By the Galerkin method, we can easily obtain the existence of solutions. Next, we prove the uniqueness of solutions.

Set $\omega=u_{1}-u_{2}$, where $u_{i}(i=1,2)$ are two solutions of (1.1)-(1.4). then $\omega$ satisfies

$$
\begin{aligned}
& \omega_{t}+\omega_{x x x}+\alpha \omega+\beta\left(u_{1} v_{1}-u_{2} v_{2}\right)+\gamma \Delta^{2} \omega=0, \\
& u_{i} v_{i}=u_{i} \int\left(u_{i}\right)_{x} \mathrm{~d} y, i=1,2, \\
& \omega(x, y ; 0)=0,
\end{aligned}
$$

Take the inner product with $\omega$, we gets

$$
\frac{1}{2} \frac{\mathrm{d}}{\mathrm{d} t}|\omega|^{2}+\alpha|\omega|^{2}+\beta\left(u_{1} v_{1}-u_{2} v_{2}, \omega\right)+\gamma|\Delta \omega|^{2}=0
$$

Furthermore 


$$
\begin{aligned}
\frac{\mathrm{d}}{\mathrm{d} t}|\omega|^{2} & \leq 2 \beta\left|\left(u_{1} v_{1}-u_{2} v_{2}, \omega\right)\right|+2 \alpha|\omega|^{2}-2 \gamma|\Delta \omega|^{2} \\
& \leq 2 \beta\left|\left(\omega \int u_{2 x} \mathrm{~d} y+u_{1} \int \omega_{x} \mathrm{~d} y, \omega\right)\right|+2 \alpha|\omega|^{2}-2 \gamma|\Delta \omega|^{2} \\
& \leq C\left(\left|\nabla u_{2}\right|_{\infty}|\omega|^{2}+\left|u_{1}\right|_{\infty}|\nabla \omega||\omega|\right)+2 \alpha|\omega|^{2}-2 \gamma|\Delta \omega|^{2},
\end{aligned}
$$

Noticing

So, we have

$$
\begin{aligned}
& |u|_{\infty} \leq C|\nabla u|^{\frac{1}{4}}|u|^{\frac{3}{4}}, \\
& |\nabla u|_{\infty} \leq C|\Delta u|^{\frac{1}{4}}|\nabla u|^{\frac{3}{4}}, \\
& |\nabla \omega| \leq C|\Delta \omega|^{\frac{1}{2}}|\omega|^{\frac{1}{2}},
\end{aligned}
$$

$$
\frac{\mathrm{d}}{\mathrm{d} t}|\omega|^{2} \leq C\left|\Delta u_{2}\right|^{\frac{1}{4}}\left|\nabla u_{2}\right|^{\frac{3}{4}}|\omega|^{2}+C\left|\nabla u_{1}\right|^{\frac{1}{4}}\left|u_{1}\right|^{\frac{3}{4}}|\nabla \omega||\omega|+2 \alpha|\omega|^{2}-2 \gamma|\Delta \omega|^{2}
$$

From Lemmas 1-3, we have

$$
\left|\Delta u_{2}\right| \leq C,\left|\nabla u_{2}\right| \leq C,\left|\nabla u_{1}\right| \leq C,\left|u_{1}\right| \leq C
$$

Using Young inequality, we obtain

$$
\frac{\mathrm{d}}{\mathrm{d} t}|\omega|^{2} \leq C|\omega|^{2}
$$

Using Gronwall inequality, we have

$$
|\omega|^{2} \leq|\omega(0)|^{2} \mathrm{e}^{2 C t}=0
$$

So, we can get $\omega=0$.

\subsection{Global Attractor}

Theorem 3.2 Assume that $f(x, y) \in H_{0}^{2}(\Omega)$ and $u_{0}(x, y) \in H_{0}^{2}(\Omega), \alpha>\frac{\beta^{2} C^{2}}{2 \gamma}$ there exists a compact global attractor $A$, such that

1) $S(t) A=A, t>0$

2) $\lim \operatorname{dist}(S(t) B, A)=0$

here, ${ }^{t} \vec{B}^{\infty}$ is a bounded set in $H_{0}^{2}(\Omega)$.

$$
\operatorname{dist}(X, Y)=\sup _{x \in X} \inf _{y \in Y}|x-y|_{E}
$$

$S(t)$ are the semigroup operators.

Proof. Let us verify theorem 2.1 conditions (1), (2), (3). In Theorem 3.2 conditions, we know that there exist the solution semigroup $S(t), E=H_{0}^{2}(\Omega), S(t): H_{0}^{2}(\Omega) \rightarrow H_{0}^{2}(\Omega)$. form Lemmas 1-3, we can get that $\forall B \subset H_{0}^{2}(\Omega)$ is a bounded set and $B$ included in the ball $\left\{|u|_{H_{0}^{2}} \leq R\right\}$,

$$
\left|S(t) u_{0}\right|_{H_{0}^{2}}^{2}=|u(x, y ; t)|_{H^{2}}^{2} \leq\left|u_{0}\right|^{2}+C_{1}|f|^{2}+C_{2}\left(t \geq 0, u_{0} \in B\right) .
$$

This shows that $S(t)(t \geq 0)$ is uniformly bounded in $H_{0}^{2}(\Omega)$. Furthermore, when $t \geq \max \left\{t_{1}, t_{2}, t_{3}\right\}$, we have

$$
\left|S(t) u_{0}\right|_{H_{0}^{2}}^{2}=|u(x, y ; t)|^{2} \leq 2\left(C_{2}+C_{3}+C_{4}\right)
$$


so, we can get that $B_{0} \geq\left\{u(x, y ; t) \in H_{0}^{2}(\Omega),|u|_{H_{0}^{2}} \leq \sqrt{2\left(C_{2}+C_{3}+C_{4}\right)}\right\}$ is bounded absorbing set of semigroup $S(t)$.

From Lemma 4, we have $|\nabla \Delta u| \leq \frac{Q}{t},(t>0), \quad\left|u_{0}\right|_{H_{0}^{2}} \leq R$. Since $H_{0}^{3}(\Omega) \rightarrow H_{0}^{2}(\Omega)$ is tightly embedded.

So the semigroup operator $S(t): H_{0}^{2}(\Omega) \rightarrow H_{0}^{2}(\Omega)$ for $\forall t>0$ is continuous.

\subsection{Dimension Estimation}

Considering the following first variation equations

$$
\begin{aligned}
& \omega_{t}(x, y ; t)+L(u(x, y ; t)) \omega(x, y ; t)=0, \\
& v(x, y ; t)=\int u_{x}(x, y ; t) \mathrm{d} y, \\
& \omega(x, y ; 0)=0, \\
& \left.\omega(x, y ; t)\right|_{\partial \Omega}=0,\left.\Delta \omega(x, y ; t)\right|_{\partial \Omega}=0
\end{aligned}
$$

where

$$
\begin{aligned}
& \omega(x, y ; 0) \in H_{0}^{1}(\Omega) \\
& L(u(t)) \omega(t)=\omega_{x x x}(t)+\alpha \omega(t)+\beta \omega_{x}(t) v(t)+\beta \int \omega_{x x}(t) \mathrm{d} y+\gamma \Delta^{2} \omega(t)
\end{aligned}
$$

It's easy to prove that the equation has a unique solution. $\omega(x, y ; t) \in L^{\infty}\left(0, T ; H_{0}^{1}(\Omega)\right)$.

Furthermore, Let $u(t)=S(t) u_{0},\left(D S(t) u_{0}\right) \omega_{0}=\omega(t), S(t)\left(u_{0}+\omega_{0}\right)=u^{*}(t)$, we can get $\forall R_{1}, R_{2}$ and $T$ are constants. There exist a constant $C=C\left(R_{1}, R_{2}, T\right)$ such that for $u_{0}, \omega_{0}$, $t$ with $\left|u_{0}\right|_{H_{0}^{1}(\Omega)} \leq R_{1}$, $\left|\omega_{0}\right|_{H_{0}^{1}(\Omega)} \leq R_{2},|t| \leq T$, we have

$$
\left|u^{*}(t)-u(t)-\omega(t)\right|_{H_{0}^{1}(\Omega)} \leq C\left|\omega_{0}\right|_{H_{0}^{1}(\Omega)}^{2},
$$

That suggests that $S(t)$ is Frechet differential at $u_{0}(x, y)$.

Let $V_{1}(t), V_{2}(t), \cdots, V_{N}(t)$ be the solutions of the linear variational equations corresponding to the initial value $V_{1}(0)=\xi_{1}, V_{2}(0)=\xi_{2}, \cdots, V_{N}(0)=\xi_{N}$. We have

$$
\frac{\mathrm{d}}{\mathrm{d} t}\left|V_{1}(t) \Lambda V_{2}(t) \Lambda \cdots \Lambda V_{N}(t)\right|^{2}-2 \operatorname{tr}\left(L(u(t)) \cdot Q_{N}\right)\left|V_{1}(t) \Lambda V_{2}(t) \Lambda \cdots \Lambda V_{N}(t)\right|^{2}=0,
$$

here $\Lambda$ represents the outer product, $\operatorname{tr}$ represents the trace, $Q_{N}$ means that the $L^{2}(\Omega)$ to the orthogonal projection on the span $\left\{V_{1}(t), V_{2}(t), \cdots, V_{N}(t)\right\}$. So, from (3.3.8) we can obtain

$$
\omega_{N}(t)=\left.\sup _{u_{0} \in A_{\xi_{n}} \in L^{2},\left\{\xi_{n} \mid \leq 1\right.} \sup _{1}(t) \Lambda V_{2}(t) \Lambda \cdots \Lambda V_{N}(t)\right|_{\Lambda_{L^{2}}^{N}} ^{2},
$$

where $\omega_{N}$ is called Secondary index, namely

$$
\omega_{N}\left(t+t^{\prime}\right) \leq \omega_{N}(t) \omega_{N}\left(t^{\prime}\right), t, t^{\prime} \geq 0
$$

so

$$
\begin{aligned}
& \lim _{t \rightarrow \infty} \omega_{N}(t)^{\frac{1}{t}}=\Pi_{n}, 1 \leq n \leq N \\
& \Pi_{n} \leq \mathrm{e}^{-q_{N}}
\end{aligned}
$$

here

$$
q_{N}=\lim _{t \rightarrow \infty} \sup \left(\inf _{u_{0} \in A} \frac{1}{t} \int_{0}^{t} \inf \left(\operatorname{tr}\left(L\left(s(\tau) u_{0}\right) Q_{N}(\tau)\right) \mathrm{d} \tau\right)\right) .
$$

Theorem 3.3 The global attractor A of Theorem 3.2 has finite fractal and Hausdorff dimension in 
$H_{0}^{1}(\Omega)$,

$$
d_{H}(A) \leq J_{0}, d_{F}(A) \leq 2 J_{0},
$$

$J_{0}$ is a minimal positive integer of the following inequality

$$
J_{0}=\frac{c-3 a+\sqrt{a^{2}+c^{2}+8 a b+2 a c}}{4 a},
$$

here

$$
a=\frac{\gamma C^{\prime}}{6}, b=\alpha+\frac{\beta}{2} C_{1}|\Delta u|_{\infty}+\frac{5}{2} C_{6}|u|_{\infty}|\Delta u|_{\infty}, c=\frac{C_{6} C^{\prime}}{2}|u|_{\infty} .
$$

Proof. From [9], we need to estimate $\operatorname{tr}\left(L(u(t)) \cdot Q_{N}\right)$ of the lower bound. Let $\varphi_{1}, \varphi_{2}, \cdots, \varphi_{N}$ be the orthogonal basis of subspace of $Q_{N} L^{2}(\Omega)$,

$$
\begin{aligned}
\operatorname{tr}\left(L(u(t)) \cdot Q_{N}\right) & =\sum_{j=1}^{N}\left\{\left(\varphi_{j x x x}+\beta \varphi_{j x} v+\beta \int \varphi_{j x x} \mathrm{~d} y+\gamma \Delta^{2} \varphi_{j}+\alpha \varphi_{j}, \varphi_{j}\right)\right\} \\
& =\sum_{j=1}^{N}\left\{\alpha\left|\varphi_{j}\right|^{2}+\gamma\left|\Delta \varphi_{j}\right|^{2}+\beta\left(\varphi_{j x} v+u \int \varphi_{j x x} \mathrm{~d} y, \varphi_{j}\right)\right\},
\end{aligned}
$$

where

So, we can obtain

$$
\left(\varphi_{j x} v, \varphi_{j}\right)=-\left(\varphi_{j}, v_{x} \varphi_{j}+v \varphi_{j x}\right)
$$

$$
\left(\varphi_{j x} v, \varphi_{j}\right)=-\frac{1}{2}\left(v_{x}, \varphi_{j}^{2}\right)
$$

Furthermore

$$
\begin{aligned}
\beta\left|\sum_{j=1}^{N}\left(\varphi_{j x} v, \varphi_{j}\right)\right| & =\frac{\beta}{2}\left|\sum_{j=1}^{N}\left(v_{x}, \varphi_{j}^{2}\right)\right| \leq \frac{\beta}{2} C\left|\sum_{j=1}^{N} \varphi_{j}^{2}\right|\left|v_{x}\right|_{\infty} \leq \frac{\beta}{2}\left|C_{1} \sum_{j=1}^{N} \varphi_{j}^{2}\right||\Delta u|_{\infty}, \\
\left|\left(u \int \varphi_{j x x} \mathrm{~d} y, \varphi_{j}\right)\right| & =\left|\left(\int \varphi_{j} \mathrm{~d} y, u_{x x} \varphi_{j}+2 u_{x} \varphi_{j x}+u \varphi_{j x x}\right)\right| \\
& =\left|\left(C_{2} y \varphi_{j}, u_{x x} \varphi_{j}+2 u_{x} \varphi_{j x}+u \varphi_{j x x}\right)\right| \\
& \leq C_{2}|u|_{\infty}\left|\left(\varphi_{j}, u_{x x} \varphi_{j}+2 u_{x} \varphi_{j x}+u \varphi_{j x x}\right)\right| \\
& \leq C_{3}|u|_{\infty}|\Delta u|_{\infty}\left|\varphi_{j}\right|^{2}+C_{4}|u|_{\infty}\left|\left(\varphi_{j}, 2 u_{x} \varphi_{j x}\right)\right|+C_{5}|u|_{\infty}\left|\left(\varphi_{j}, u \varphi_{j x x}\right)\right|, \\
\left(\varphi_{j}, 2 u_{x} \varphi_{j x}\right)= & -2\left(\varphi_{j x} u_{x}+\varphi_{j} u_{x x}, \varphi_{j}\right)=-2\left(\varphi_{j x} u_{x}, \varphi_{j}\right)-2\left(u_{x x}, \varphi_{j}^{2}\right)
\end{aligned}
$$

hence

$$
\begin{aligned}
& \left(\varphi_{j}, 2 u_{x} \varphi_{j x}\right)=-\left(u_{x x}, \varphi_{j}^{2}\right), \\
& \left(\varphi_{j}, u \varphi_{j x x}\right)=-\left(\varphi_{j x} u, \varphi_{j x}\right)+\frac{1}{2}\left(\varphi_{j}^{2}, u_{x x}\right),
\end{aligned}
$$

Taking (3.3.15)-(3.3.16) into (3.3.14), we can get

$$
\left|\left(u \int \varphi_{j x x} \mathrm{~d} y, \varphi_{j}\right)\right| \leq C_{6}|u|_{\infty}\left(\frac{5}{2}|\Delta u|_{\infty}\left|\varphi_{j}^{2}\right|+\left|\nabla \varphi_{j}^{2}\right||u|_{\infty}\right),
$$

Set $\lambda_{j}, j=(1,2,3, \cdots)$ are eigenvalues of $-\Delta u=\lambda u$ and $\varphi_{j}$ are the corresponding eigenfunctions. Satisfying

$$
\left|\nabla \varphi_{j}\right|^{2}=\lambda_{j},\left|\Delta \varphi_{j}\right|^{2}=\lambda_{j}^{2},\left|\varphi_{j}\right|^{2}=1, \lambda_{j} \geq\left[\frac{(j-1)^{\frac{1}{2}}}{2}-1\right]^{2} \sim C^{\prime} j,
$$

so, we can get 


$$
\operatorname{tr}\left(L(u(t)) \cdot Q_{N}\right) \geq \gamma \sum_{j=1}^{N} \lambda_{j}^{2}-N \alpha-\frac{\beta}{2} N C_{1}|\Delta u|_{\infty}-\frac{5}{2} C_{6} N|u|_{\infty}|\Delta u|_{\infty}-C_{6}|u|_{\infty}^{2} \sum_{j=1}^{N} \lambda_{j},
$$

Let

$$
a=\frac{\gamma C^{\prime}}{6}, b=\alpha+\frac{\beta}{2} C_{1}|\Delta u|_{\infty}+\frac{5}{2} C_{6}|u|_{\infty}|\Delta u|_{\infty}, c=\frac{C_{6} C^{\prime}}{2}|u|_{\infty},
$$

when

$$
N>\frac{c-3 a+\sqrt{a^{2}+c^{2}+8 a b+2 a c}}{4 a}
$$

we have

$$
\operatorname{tr}\left(L(u(t)) \cdot Q_{N}\right)>0
$$

so, we can obtain

$$
d_{H}(A) \leq J_{0}, d_{F}(A) \leq 2 J_{0}
$$

\section{Funding}

This work is supported by the National Natural Sciences Foundation of People's Republic of China under Grant 11161057.

\section{REFERENCES}

[1] G. R. Sell, "Global Attractors for the Three-Dimensional Navier-Stokes Equations," Journal of Dynamics and Di_erential Equations, Vol. 1, 1996.

[2] J. K. Hale, “Asymptotic Behaviour of Dissipative Systems," Mathematical Surveys and Monographs, Vol. $25,1988$.

[3] G. Sell and M. Taboada, "Local Dissipativity and Attractors for the K-S Equation in Thin 2D Domains," Journal of Nonlinear Analysis, Vol. 7, 1992.

[4] R. Temam and S. Wang, "Inertial Forms of Navier-Stokes Equations on the Sphere," Journal of Functional Analysis, Vol. 2, 1993.

[5] B. L. Guo and Y. S. Li, “Attractor for Dissipative Klein-Gordon-Schrdinger Equations in R3," Journal of Differential Equations, Vol. 2, 1997.

[6] X. Y. Du and Z. D. Dai, "Global Attractor of Dissipative KDV Equation about Cauchy Problem," Acta Mathematica Scientia, Vol. 20, 2000.

[7] L. X. Tian, Y. R. Liu and Z. R. Liu, "The Narrow 2D Weak Local Attractor for Damped KDV Equation," Applied Mathematics and Mechanics, Vol. 21, 2000.

[8] B. L. Guo, "Method of Vanishing Viscosity and Viscosity Difference Format," Science Press, Beijing, 1993.

[9] G. G. Lin, "Nonlinear Evolution Equation,” Yunnan University Press, Yunnan, 2011. 